\title{
ELECTRICIDAD Y CRECIMIENTO ECONOMICO. UNA HIPOTESIS DE INVESTIGACION
}

FRANCESCA ANTOLIN

Universidad de Barcelona

\section{Introducción}

Para centrar la polémica empezaré con una metáfora. Nadie puede dudar de que el teléfono era una herramienta corriente en la vida española de 1970. Sin embargo, cuando en aquel año me fui a vivir a los EE. UU. pude entender vívidamente que la penetración de las comunicaciones telefónicas en Es. paña resultaba enormemente superficial, si se la comparaba con su papel en la vida americana $y$, sobre todo, en las actividades comerciales. $Y$ todo esto no era idiosincrático, no se debía a que a los americanos les gustase más hablar por teléfono que a nosotros, sino que era resultado de la calidad y del precio del servicio en uno y otro país. Incluso sin hablar de precios, en España costaba años obtener nuevas líneas, y en los EE. UU. costaba un solo día. Como consecuencia de ello, ninguna empresa sensata de nuestro país podía basar su negocio en un instrumento que, en cambio, los americanos podian tomar como un dato. Esto no quiere decir que el teléfono no fuese importante en España. Pero, por difícil que resulte cuantificar la diferencia, cualquiera que sepa de qué hablo deberá reconocer que su papel en uno y otro país no tenían nada que ver. Por otra parte, el origen de esta diferencia podía atribuirse esencialmente a una causa: los muy distintos márgenes de maniobra con que se movían las compañías productoras, por mucho que ambas fueran monopolistas (la elección de 1970, aparte de autobiográfica, se justifica porque la estructura de la industria telefónica estadounidense ha cambiado mucho desde entonces). Aunque monopolio, la Bell estaba sometida a múltiples presiones, a una regulación efectiva, mientras que la posibilidad de que una empresa no pudiese trasladarse durante meses por falta de teléfono, por ejemplo, no inmutaba en lo más mínimo entonces, ni posiblemente ahora, a nuestra compañía nacional. Recurro a este ejemplo para señalar que, a mi entender, Sudrià y yo no estamos tan en desacuerdo como él parece creer, sino que estamos interesados en aspectos distintos de una misma realidad. Aprovechando el ejemplo de los teléfonos, yo diría que a él le interesaría destacar que en 
España se extedieron rápidamente. Pero yo insistiría en que los existentes servían para mucho menos de lo que hubieran podido, en que podría haber habido más y mejor utilizados, que vale la pena tener en cuenta la calidad y los precios relativos del servicio a la hora de explicar estas diferencias, y que la hipótesis de un monopolio mal regulado como causa de ineficiencias parece atractiva para empezar. Todo esto en metáfora. A partir de ahora, hablemos de electricidad.

Si no lo entiendo mal, el propósito de Sudrià en su réplica es reafirmar la validez de ciertas tesis y líneas de argumentación propuestas por él mismo y por Jordi Maluquer acerca de los inicios de la electrificación en España, a las que, en su opinión, contradigo en mi artículo de 1988 en esta REvisTA. Para ello presenta nueva evidencia empírica, después de descalificar la seleccionada en mi artículo. Mi objetivo en esta nota no es poner en cuestión las nuevas cifras ni las conclusiones que extrae de ellas. Lo que quiero es explicar qué preguntas me hacía en aquel artículo, por qué las respuestas parciales a las que llegué me fueron útiles en trabajos posteriores, y defender el uso que hice entonces de los datos empleados, en relación a aquellas cuestiones. Por el camino procuraré aclarar los términos de la supuesta discrepancia, reflexionar sobre los límites de la documentación cuantitativa disponible y sobre el tipo de cuestiones, en gran parte no resueltas, que parecen relevantes para el estudio del sector eléctrico español.

\section{Sobre propósitos y conclusiones}

Insisto en que no creo que tengamos «dos visiones contrapuestas sobre las primeras fases de la electrificación en España», simplemente porque el objeto de mi trabajo no es responder a la misma pregunta que le interesa a Sudrià. Para él, parece esencial mantener que la electrificación fue rápida y que ello implicó «uno de los pocos ejemplos de aprovechamiento rápido y profundo de una innovación tecnológica que podemos hallar en la historia económica de la España contemporánea». En cambio, mi propósito en el artículo en cuestión, como su título indica, es investigar la posibilidad, o no, de establecer una conexión entre electrificación y crecimiento económico en España.

Para ello: a) empiezo seleccionando «algunas tesis sobre la contribución de la electricidad al crecimiento económicos ', y b) destaco los mecanismos que, según los autores consultados, activan los procesos de electrificación. En la segunda parte recojo los datos disponibles para el caso español que creo pertinentes para: c) realizar «un primer contraste de hipótesis respecto a las causas

' Los énfasis son de ahora. 
económicas que marcan el ritmo y el grado de extensión de la electricidad en el sector industrial español».

Las conclusiones de dicho ejercicio son que «en España parece cumplirse mejor la hipótesis de Woolf ${ }^{2}$. Son las ventajas de precios relativos entre electricidad, carbón y trabajo, más que las ventajas puramente tecnológicas, según la hipótesis de Devine ${ }^{3}$, las que activan el proceso de electrificación y abren las puertas a una mayor capitalización de la economía industrial» (p. 648). En este sentido compruebo que resulta coherente relacionar la evolución de los precios relativos con el ritmo e intensidad del proceso electrificador en nuestro país. Un primer test nos lo proporciona el País Vasco, donde se observa que a un distanciamiento creciente entre los precios de los inputs energéticos según provincias (véase Apéndice) corresponde un nivel de electrificación mayor, en el sector de industria pesada que tenemos documentado (pp. 647-648). Un segundo test de consistencia en favor de la hipótesis de que los precios relativos son importantes como estímulo a la electrificación nos lo ofrece el caso catalán. En este sentido destaco que, en Cataluña, "con un elevado consumo de energía por habitante, el carbón fue siempre más caro que en Bilbao y la electricidad más barata, porque se introdujo en condiciones de fuerte competencia. En varios trabajos 4 . J. Maluquer muestra la aceleración que experimenta la producción de electricidad en el período de la guerra y la casi total sustitución del vapor por la electricidad en ciertos sectores de industria ligera, predominante en Cataluña» (p. 645). Comparo después el caso español con el de otros países. Para ello, acepto los datos de aquellos autores que teorizan sobre la conexión entre electrificación y crecimiento económico en los EE. UU., y que destacan mecanismos de conexión entre una y otro. Observo que en nuestro país se da la misma relación causal a contrario: "en nuestro caso, a un distanciamiento muy modesto entre precios relativos le corresponde un proceso de avance de la electrificación muy lento respecto al vapor y poco radical en cuanto a la estructura interna del sector productor» (p. 648).

Finalmente, constato que la debilidad relativa de los estímulos a la electrificación y a la capitalización, a la que apunta la documentación fragmentaria disponible, es congruente con «lo que sabemos respecto al comportamiento del sector energético e industrial español». Efectivamente, «en nuestro pasado industrial no se da la conexión apuntada por Devine entre electrificación y crecimiento económico. Mientras que en EE. UU. la adopción generalizada de la electricidad es simultánea al ahorro de energía por unidad del PNB y al incremento en la productividad del trabajo y del capital, Sudrià observa que

\footnotetext{
2 Woolf (1984).

'Devine (1983).

- Maluquer (1984 y 1987).
} 
en el caso español la ratio consumo de energía/PNB es siempre creciente ${ }^{5}$. $Y$ Carreras estima un descenso en la productividad del trabajo industrial en las primeras décadas del siglo ${ }^{\circ}$. La productividad del trabajo no remonta el nivel de 1900 hasta la década de 1960, mientras que, en el conjunto español, la electricidad seguramente superaría al vapor una vez finalizada la Guerra Civil» (pp. 646 y 648).

En definitiva: se ha propuesto que la introducción de la electricidad puede ser en sí misma, vía mecanización e incremento en la productividad, un factor muy favorable al desarrollo. Dado el escaso auge que parecen haber tomado dichas manifestaciones en España durante el período considerado, o bien el grado de electrificación fue débil, o bien la citada conexión es discutible. Quienes, como Woolf, la discuten incluso en el caso americano, señalan que la principal razón de su introducción no estriba en una ventaja tecnológica abrumadora, sino más bien en una ventaja económica, de precios relativos. Mi constatación de que los precios relativos entre electricidad y energías alternativas no era especialmente favorable no daba respuesta concluyente a la cuestión del nexo entre electrificación y desarrollo, pero era coherente no con una, sino con dos posibilidades. Ya que, por un lado, si tal nexo existiese, mis observaciones eran coherentes con un bajo grado de electrificación (sobre las posibilidades de medirlo volveré más adelante). $Y$, si no existiese relación causa-efecto, seguia siendo interesante contrastar la hipótesis de Woolf según la cual el estímulo a electrificar es más económico que tecnológico y, por tanto, más débil allí donde las ventajas relativas de precios son menores, aunque las tecnologías sean universalmente disponibles. Desde este punto de vista, creo que quienes tienen algo pendiente son quienes ven en España un ejemplo de brillante electrificación no acompañada de desarrollo.

Por mi parte, me sentí más atraída hacia otras cuestiones que quedaban abiertas, de ser acertados mis primeros contrastes. Concluido aquel primer test, "centrado en el análisis de precios relativos, al tratar de entender un proceso de sustitución de factores» (p. 649), en trabajos posteriores busqué posibles explicaciones a dos fenómenos que los datos disponibles parecían sugerir. El primero es el escaso distanciamiento relativo entre los precios de electricidad, carbón y trabajo, en comparación a los EE. UU. y, sobre todo, en relación a lo que hubiese podido esperarse, dado el contexto en que se produjo la introducción de la electricidad en España ${ }^{7}$. En efecto, el carbón fue muy caro para casi todas las actividades industriales a lo largo del xIX (lo seguiría siendo en el $\mathrm{xx}$ ), y disponíamos de recursos hidráulicos abundantes, de lo que eran bien conscientes los contemporáneos. Además, en un trabajo anterior ya

Sudrià (1987).

- Carreras (1987).

' Antolín (1989 a). 
habia visto que, desde los inicios, la industria eléctrica, al menos en el País Vasco, dispuso de iniciativa empresarial, capital y tecnología de punta comparables a los de los países industriales avanzados, por lo que se habría justificado una oferta de electricidad muy competitiva en precios y una rápida sustitución de inputs energéticos. Esta última consecuencia esperada contrastaba, en cambio, con el segundo fenómeno que había observado: la, a mi entender, moderada penetración de la electricidad y la estructura interna del sector productor observada.

En los trabajos que siguieron a mi artículo en esta REvista observo como, en ausencia de participación directa del Estado en su creación y a falta de una regulación significativa de las empresas del sector hasta después de la Guerra Civil, unas cuantas empresas eléctricas, relacionadas a través de holdings, se reparten los mejores mercados en los que actúan como monopolistas ${ }^{8}$. Esta circunstancia permite una explicación coherente del primer fenómeno observado: el moderado distanciamiento en los precios. Dada la estructura de mercado en que actúan, las características de la demanda y del bien que suministran, las eléctricas están en condiciones de aplicar una política de discriminación de precios que les permite situar el precio de la electricidad comercial a escasa distancia por debajo del de las energías alternativas, y obtener las rentas derivadas de aquellas potenciales ventajas de coste. $\mathrm{Y}$ también los escasos ejem. plos de situaciones en que prevalece la competencia, las documentadas por Maluquer y García de la Infanta para el área industrial de Barcelona y para Madrid durante períodos de tiempo bien determinados, corroboran la misma explicación a contrario ${ }^{9}$. En efecto, en tales casos los precios de la electricidad se reducen en mayor proporción que los de las energías alternativas, y ello resulta en una rápida adopción de la electricidad.

La segunda observación, la lentitud en la sustitución de energías alternativas y en la concentración de sector productor (no sólo de la electricidad comercial, sino también de la producida por autoproductores), admite una explicación coherente atendiendo a varias razones: a) consideraciones de costes relativos entre la eléctrica y demás energías alternativas; $b$ ) el marco temporal en que se inscribe la política de maximización de beneficios de las eléctricas, que no siempre les lleva a adoptar tecnologías o políticas de precios que serían óptimas en ausencia de inversiones anteriores y atendiendo sólo a consideraciones de corto plazo; y c) las características de la demanda de energía, que depende de la tradición industrial, el ritmo de crecimiento del sector y de las posibilidades e incentivos para la mejora de la productividad ${ }^{10}$.

- Antolín (1988 b).

- Maluquer (1983) y García de la Infanta (1986).

10 El lector interesado puede referirsc a mi artículo «Un servicio público con escasa 
De lo que llevo dicho se deduce que mi primer trabajo sobre electricidad, y lo mismo puedo decir de los que le siguieron, no es un proyecto de historia comparativa, ni cuantitativa, sino un primer test en el que utilizo los datos disponibles para España al objeto de: $a$ ) identificar los mecanismos incentivadores de la electrificación; $b$ ) comparar su comportamiento y consecuencias respecto a la experiencia analizada por los autores americanos, de los que tomo la metodología aplicada, y, fundamentalmente, $c$ ) diseñar hipótesis de trabajo a corroborar más tarde. Dicha estrategia de investigación es coherente con mi observación en el Apéndice de aquel artículo respecto a los datos disponibles, su significación y posibilidades, y en esto creo estar de acuerdo con Sudrià: los datos disponibles respecto a la electrificación en general y la española en particular son fragmentarios, miden magnitudes de naturaleza distinta según sectores y períodos, y se extraen de contextos económicos con condiciones de oferta y demanda de energía diversas, por lo que no deben tomarse como más de lo que son, evidencia parcial. En mi opinión, su utilización debe restringirse al propósito de provocar y formular hipótesis, pero no pueden servir para su ratificación, como parece querer Sudrià, ni, por tanto, convertirse en el centro de la polémica. Si ésta debe existir, pienso que debería centrarse en elementos más cualitativos, como el análisis de los factores que condicionan la conducta de productores y consumidores. Por ejemplo, me gustaría saber qué hay de incoherente en proponer que, a igualdad de posibles costos de producción, una estructura esencialmente monopolística tienda a mantener los precios de la electricidad relativamente altos y, con ello, contenga las posibilidades de penetración de esta industria por debajo de las que hubieran sido posibles en situaciones más competitivas o más estrictamente reguladas.

\section{Sobre la utilización de datos}

Sudrià discrepa respecto al cálculo de la magnitud de distanciamiento de los precios relativos entre carbón y electricidad y sobre el ritmo de la electrificación en España. Creo que, según su interpretación, lo que falla en dichos cálculos no es tanto la utilización que yo hago de los datos españoles, sino sobre todo la utilización que de sus propios datos hacen los autores americanos que yo cito. Como no he pretendido ni pretendo ser una especialista en temas energéticos èstadounidenses, no me corresponde defender la utilización de datos y las conclusiones a las que llegan los investigadores de aquel país.

intervención. Los primeros cuarenta años de electricidad en España», Economia Industrial, julio-agosto 1988. 
Sólo quiero afirmar la oportunidad de haber tomado sus cifras, especialmente tratándose de documentación sobre precios energéticos, sobre cuyas limitaciones coincidimos Sudrià y yo, especialmente a la hora de establecer comparaciones. Los datos rebatidos por Sudrià sobre precios relativos entre carbón y electricidad en EE. UU. son los que selecciona Woolf en el contexto de un amplio proyecto de investigación, publicado en una de las revistas más exigentes respecto al rigor cuantitativo, y precisamente para medir los niveles de su principal factor explicativo. No son una aproximación y sí se indica el tipo de consumidores y la fuente que los contiene: son datos del Twentieth Century Fund ${ }^{11}$ referidos al consumo doméstico ${ }^{12}$. Woolf, que analiza la incidencia del precio sobre el comportamiento de la elasticidad de sustitución entre factores productivos (entre ellos carbón y electricidad) para 15 sectores industriales en el período 1909-1929, con datos de las Historical Statistics y el Census of Manufactures, toma dicha selección como el indicador más conservador en la tendencia a disminución de los precios de la electricidad en dicho país ${ }^{13}$. Sin recurrir a argumentos de autoridad, me pareció prudente aceptar que dicho tratamiento ofrecía garantías de haber sido seriamente contrastado.

Está claro que la evolución de los precios relativos refleja cuánto se distancian los precios de dos artículos en un período determinado. Naturalmente que sería interesante contemplar el distanciamiento de los precios desde el momento en que los dos inputs energéticos empiezan a competir para una misma gama de usos. A falta de datos completos, en el apéndice de mi artículo ofrezco indicaciones sobre su trayectoria previsible. Sobre las limitaciones asociadas con la utilización de índices, no puedo tomar como crítica dirigida a mí sino en todo caso como lamentación conjunta las reservas de Sudrià, porque él también los utiliza. Coincido, por otro lado, en que seria bueno poder comparar precios reales, aunque para muchas consideraciones basta con los relativos. Pero si, en general, ya es un problema complejo definir qué se entiende por un precio real, en el caso de comparar diversas fuentes de energía habría que ajustar atendiendo a la eficacia de cada uno de los conversores, que además de ser específicos a cada tipo de input energético son también susceptibles de evolucionar en función de consideraciones de costes. No me parece, pues, que vayamos a avanzar mucho por ahi.

Para obtener indicadores sobre la transición del vapor a la electricidad, proceso sobre el que espero aportar nueva evidencia a partir de una investigación actualmente en curso, utilizo los escasos datos disponibles en el País Vasco, correspondientes a sectores industriales que, como he dicho

"Twentieth Century Fund (1976).

12 Woolf (1983), p. 178.

13 Woolf (1983), p. 178, nota 3. 
en un trabajo más detallado para dicha zona, están lejos de ser representativos ${ }^{14}$. En ellos se ajusta la cifra de potencia instalada siguiendo la recomendación hecha por Devine ${ }^{15}$, y otros autores según los cuales tan sólo entre un séptimo y un quinto de la potencia eléctrica instalada puede considerarse activa cuando se trata de establecer comparaciones con el vapor ${ }^{16}$. Desde luego, doy por supuesto que los cálculos de Devine sobre la transición en Estados Unidos son consistentes con su propia observación.

Respecto al grado de concentración del sector productor, la evidencia que presento sobre la potencia de las instalaciones eléctricas del País Vasco y el tipo de productores tiene la virtud de incluir información más completa, sin llegar a ser exhaustiva, que la contenida en publicaciones oficiales que se manejan a menudo, como el Anuario Estadístico de España o la de la Cámara Oficial de Productores y Distribuidores de Electricidad. Quiero insistir en que los datos de dicho cuadro 5 (pág. 646) muestran un nivel de concentración del sector productor muy moderado, después de casi cincuenta años de introducción de la industria eléctrica y no dos décadas. Escasa concentración respecto al sector productor americano sobre el que sí aporto documentación, y del que se puede afirmar que, dado que mayoritariamente obtiene la electricidad de fuentes térmicas, disponía de tecnologías de producción de menor escala, por lo que debe esperarse un nivel de concentración menor ". Si bien lo más llamativo y significativo de la situación vasca es el modesto nivel de concentración alcanzado dados los supuestos de partida en términos de oferta y demanda de energía en dicha zona. Teniendo en cuenta las características del mercado eléctrico local, en mi artículo de 1989 explico el nivel de integración alcanzado en el sector como resultado de la estrategia de producción y precios de Hidroeléctrica Ibérica, la empresa que controla el subsector de electricidad comercial. Desde su constitución en 1901, dicha empresa actúa como monopolio escasamente regulado en el suministro de electricidad, y como tal selecciona, atendiendo a criterios de costes y remuneración, las áreas de mercado y los clientes dentro de éstas que le resultan más convenientes en cada momento. El resto de productores, los pequeños suministradores y los autoproductores surten la demanda no satisfecha por Hidroeléctrica, la ubicada en áreas de mercado con demandas dispersas o poco intensas y, en el caso de autoproductores, las que pueden cubrirse a un coste inferior al que resulta de comprar electricidad comercial.

En este aspecto, y teniendo en cuenta que los datos utilizados por Sudrià se refieren a las empresas mayores del sector de electricidad comercial, las

14 Antolín (1989 b).

is Devine (1983), p. 362.

16 Du Buff (1987) y Daugherty (1933).

"Antolín (1989 b), p. 126. 
que estaban asociadas a la Cámara Oficial de Productores y Distribuidores de Electricidad, organismo que elabora a las estadísticas disponibles para el conjunto español en este período, lo que consigue probar es la estructura de monopolio del sector de electricidad comercial. Dicha estructura, en ausencia de regulación por parte del Estado, es la responsable, a mi entender, de que la electricidad comercial suministre tan sólo a una selección de los consumidores potenciales, los que están dispuestos a pagar precios elevados, y ello explica, en última instancia, la escasa concentración del conjunto del sector productor.

Como he dicho, no es mi propósito en estas líneas discutir los nuevos datos aportados por Sudrià en defensa de sus tesis. Lo que quiero destacar es que, por el camino, obtiene conclusiones que me parecen una clara réplica a las tesis sostenidas hasta ahora por los especialistas en temas de energía. Teniendo en cuenta que España no experimenta aumentos en la productividad, ni tasas de crecimiento comparables a las de los paises industriales avanzados, de acuerdo con los hallazgos de Sudrià, y en la medida en que «la electrificación fue más intensa que la de otros paises más avanzados, incluidos los Estados Unidos". el caso español sería un contraejemplo a las tesis sostenidas por muchos sobre la conexión entre electricidad y crecimiento económico, y a ellos debería dirigir la réplica.

\section{Algunos comentarios finales}

Si creemos que la electricidad fue un estímulo importante al crecimiento económico, hay que explicar por qué en nuestro país no se da dicha conexión. La tesis enunciada y sostenida por los historiadores económicos que estudian el comportamiento del sector industrial sobre la contribución de la electricidad al crecimiento económico debe considerarse, como toda tesis, a varios niveles de detalle: en su enunciado general y, sobre todo teniendo en cuenta la letra pequeña, en las hipótesis que deben concurrir para que aquélla se cumpla. En el caso de la electricidad, las hipótesis se refieren a las circunstancias en las que se realiza la adopción de la nueva tecnología eléctrica. Dichas circunstancias se pueden diferenciar, para su mejor estudio, en varias etapas: la generación de electricidad, su comercialización y su consumo.

1) Sobre la fase de producción.-No es suficiente con tener recursos: hay que poder y saber utilizarlos. En España predomina el monopolio local en el sector de producción hidroeléctrica. Hay barreras a la entrada que dificultan la competencia (se concentran las concesiones sobre utilización de 
saltos de agua en manos de unos pocos), y, además, los productores actúan a veces de acuerdo con estrategias de maximización a corto plazo. Esta estructura no es mala en sí misma para un sector como el eléctrico, pero si se acepta debe acompañarse de una regulación efectiva.

2i Sobre la fase de comercialización.- No es lo mismo el coste de generación que el precio de venta. Creo que Sudrià confunde repetidamente la existencia de recursos hidráulicos abundantes y de posibles bajos costes de generación con que los consumidores obtengan la energía a precios más baratos. Creo que esto es cierto si y sólo si ningún agente se apropia de las rentas antes de que lleguen al consumidor, mientras que a mí me ha interesado quién y cómo se las apropia. Al evaluar los resultados de una industria con características de monopolio natural debe tenerse en cuenta la estructura del sector y el marco institucional en la que actuán los productores. A lo mejor, el caso de la electricidad, para el que estamos tan bien predispuestos, se puede utilizar para generar hipótesis sobre aspectos que diferencian a los países avanzados de los países seguidores en la utilización de tecnologías dis. ponibles para todos: la organización de mercado del sector productor y la política del Estado respecto a éste.

3) Sobre la fase de consumo.- No es lo mismo estar conectado a la red de suministro eléctrico que obtener todas las ventajas potenciales de la electricidad. Para esto es preciso que la electricidad sea abundante y de calidad (suministro fiable). Su precio debe ser suficientemente bajo y los consumidores deben estar motivados para realizar inversiones que lleven a la adopción de las nuevas estructuras de producción que propicia la electricidad. Así, un país puede estar muy «electrificado», tener la mayoría de los consumidores de energía abonados a la electricidad y simultáneamente sacar poco rendimiento de esta innovación. En España sabemos que, por lo menos, éste fue el caso en el sector de consumo doméstico. Lo importante es ver si en España se dieron las últimas consecuencias esperadas de la electrificación. $\mathrm{La}$ investigación debe seguir intentando cuantificar aspectos formales, pero sobre todo se trata de esclarecer el marco de análisis, las hipótesis que sub. yacen a las proposiciones teóricas y su manifestación concreta en un escenario determinado. 


\section{REFERENCIAS}

Antolín, F. (1988 a): «Electricidad y crecimiento económico. Los inicios de la electricidad en Españan, Revista de Historia Económica, año VI, núm. 3.

- (1988 b): “Un servicio público con escasa intervención. Los primeros cuarenta años de electricidad en Españam, Economia Industrial, julio-agosto.

- (1989 a): «Electricity and Economic Growth. A view from Spain», Actas del Secont World Congress of the Cliometrics Society.

- $(1989 b)$ : «Hidroeléctrica Ibérica y la electrificación del País Vasco», Economia Pública, $5,4$.

Carreras, A. (1987): "La industria: atraso y modernización», en J. Nadal, A. Carreras y C. Sudrià (eds.), La economia española en el siglo XX, Ariel.

Devine, W. (1983): «From Shafts to Wires: Historical Perspectives on Electrification», Journal of Economic History, 43 (2).

DAugherTy, C. R. (1933): «Horsepower Equipment in the United States, 1869-1929», American Economic Review, XXIII.

Du BofF, R. B. (1967): «The Introduction of Electric Power in American Manufacturing», Economic History Review, vol. XX, núm. 3.

GARCía de LA INFANTA, J. M. (1986): Los primeros pasos de la luz eléctrica en Madrid y otros acontecimientos, Fondo Natural, Madrid.

Mnluquer, J. (1983): «L'Electricitat», en J. Nadal et al., Producció $i$ consum d'energia en el creixement economic modern: el cas catald (inédito).

- (1987): «L'électricité, facteur de développement en Espagne, 1900-1936\%, en F. Cardor (ed.), Un siècle d'électricité dans le monde, 1880-1980, Presses Universitaires de

France.
SUDRì, C. (1987): «Un factor determinante: la energía», en J. Nadal, A. Carreras y C. Sudrià (eds.), La economia española en el siglo XX, Ariel.

Woolf, A. (1984): "Electricity, Productivity, and Labor Saving in American Manufac- . turing, 1900-1929», Explorations in Economic History, 21, abril. 